\title{
GIARDIA CATI R. DESCHIENS, 1925, DU CHAT DOMESTIQUE (FELIS DOMESTICA)
}

\author{
Par R. DESCHIENS
}

L'infection spontanée du chat domestique par Giardia a été signalée par Grassi (1881), Grassi et Schewiakoff (1888), Janowski (1897), Wenyon et O'Connor (1917), Brumpt (communication orale), Doflein (1919), Gordon et Young (1922), Hogue et Van Winckle (1923). En outre, Hegner (1924) a relevé un cas d'infection spontanée du lynx rouge d'Amérique (Lynchus rufus Gubdens) par une giardie probablement différente d'ailleurs de celle du chat domestique.

Lorsqu'on se rapporte aux travaux de ces auteurs, on constate qu'il n'existe pas d'étude méthodique des formes végétatives ou des kystes de Giardia observés chez le chat. Chez le lynx rouge, Hegner a étudié la structure des kystes de giardies, mais il n'a pas eu l'occasion d'observer les formes végétatives.

J'ai présenté à la Société de Biologie (Bulletin distribué le 16 mai 1925), une note dans laquelle la giardie du chat est définie morphologiquement et biologiquement et décrite sous le nom de Giardia cati n. sp. Quelques jours plus tard, R.-W. Hegner, le savant protozoologiste de Baltimore (U. S. A.) a publié dans The American Journal of Hygiene, de mai 1925, numéro distribué en juin 1925 (1), une note relative à la morphologique de la giardie du chat qu'il décrit sous le nom de Giardia felis n. sp. Signalons dès maintenant que Giardia cati et Giardia felis paraissent identiques ; s'il en est bien ainsi, Giardia felis doit tomber en synonymie.

Dans ce mémoire, annoncé à la Société de Biologie, et dont la publication a été retardée afin de tenir compte de la note de Hegner, je développe d'abord, l'étude morphologique et expérimentale de la giardie du chat: Giardia cati; j'établis ensuite les arguments sur lesquels se fonde la validité de cette espèce, puis je fais une étude comparée de Giardia cati et de Giardia felis.

J'ai eu à ma disposition une chatte adulte spontanément infectée par Giardia et présentant des alternances de transit intestinal

(1) Cette date m'a été confirmée par le professeur R.-W. Hegner dans une lettre du 21 juillet 1925 .

Annales de Parasitologie, T. IV, $\mathrm{x}^{\circ} 1 .-1 \mathrm{e}$ janvier 1926, p. 33-48.

3. 
normal et de diarrhée. Pendant les poussées de diarrhée, on notait la présence de kystes de Giardia dans les selles, en médiocre quantité d'ailleurs ; 8 à 10 par préparation à frais pour une lamelle de $22 \times 22$.

Des matières fécales provenant de cette chatte, recueillies en période diarrhéique, et contenant 8 à 10 kystes de Giardia vivants (épreuve de l'éosine) par préparation, ont été ingérées à la dose de deux grammes par un jeune chat de 2 mois (chat $n^{\circ} 1-1$ ), pris parmi trois animaux reconnus non infectés par Giardia, après un examen méthodique quotidien pendant 21 jours. Deux chats de même âge, ayant cohabité avec le précédent jusqu'au jour de l'inoculation, ont été conservés comme témoins.

Снат N ${ }^{\circ} 1-1$. - Le 5 juin 1924 : Inoculation " per os " avec des kystes vivants de giardies (souche chat). Du 5 au 12 juin, selles normales ou para-normales, Giardia (-). Le 13 juin, diarrhée avec présence de rares formes végétatives et de nombreux kystes de Giardia, dans les selles. Le 14 juin, l'animal est sacrifié (chloroforme) ; l'autopsie pratiquée immédiatement après la mort indique la répartition topographique et quantitative suivante des giardies dans l'intestin.

\begin{tabular}{|c|c|c|c|c|c|}
\hline Estomac & DUOdÉNuM & $\begin{array}{l}\text { TIERS SUpÉRIEUR } \\
\text { DE L'INTESTIN } \\
\text { GRÈLE }\end{array}$ & Trers MOYEN & $\begin{array}{c}\text { TIFRS } \\
\text { INFÉriEUn }\end{array}$ & $\underset{\text { INTESTIN }}{\text { GRos }}$ \\
\hline$(-)$ & $\begin{array}{c}\text { Formes vég. } \\
(+)\end{array}$ & $\begin{array}{c}\text { Formes végét. } \\
\qquad(+++)\end{array}$ & $\begin{array}{c}\text { Formes vég. } \\
(+t)\end{array}$ & $\begin{array}{c}\text { Kystes } \\
\text { rares }\end{array}$ & $\begin{array}{c}\text { Kystes } \\
(++)\end{array}$ \\
\hline
\end{tabular}

Les animaux témoins sont normaux.

Des frottis de formes végétatives ont été fixés au Bouin colorées par l'hématoxyle ferrique et montés au Baume.

Снат N 2-1. - L'un des deux témoins est inoculé par ingestion de deux grammes de selles provenant du chat $n^{\circ} 1-1$, riches en kystes vivants de Giardia. L'animal est sacrifié le onzième jour et l'autopsie révèle des formes végétatives de giardies, du duodénum au tiers inférieur de l'intestin grêle, des kystes et quelques formes végétatives dans le gros intestin. Frottis fixés au Bouin, coloration par l'hématoxyle ferrique, montage au baume comme pour le chat $\mathrm{n}^{\circ} 1-1$.

Le témoin sacrifié et autopsié le dernier ne présentait pas de Giardia. Le chat $\mathrm{n}^{\circ} 1-1$ et le témoin étaient infectés par Dipylidium caninum. 


\section{Etude morphologique}

Nous donnons tout d'abord, dans le tableau suivant, les mensurations générales, portant sur 100 exemplaires, avant d'étudier successivement les différents types morphologiques observés.

Tablead 1: 100 exemplaires mesurés (1)

\begin{tabular}{|c|c|c|c|c|c|c|c|c|}
\hline \multicolumn{3}{|c|}{ LONGUEUR : L } & \multicolumn{3}{|c|}{ LARGEUn: $l$} & \multicolumn{3}{|c|}{$\begin{array}{l}\text { RAPPORT DE LA LONGUEUR } \\
\text { A LA LARGEUR }\end{array}=\frac{\text { L }}{l}$} \\
\hline $\begin{array}{l}\vdots \\
\vdots \\
\vdots \\
\vdots \\
\vdots\end{array}$ & 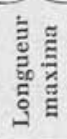 & ह & 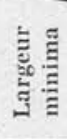 & 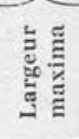 & 嘅 & 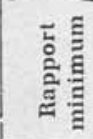 & 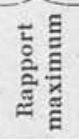 & ह气 \\
\hline $11 \mu$ & $16 \mu$ & $13 p$ & $6 \%$ & $9 \varphi, 5$ & $7 \mu, 5$ & 1,5 & 2,16 & 1,76 \\
\hline
\end{tabular}

Si, au lieu d'exprimer le maximum de fréquence, on prend la moyenne des mensurations, les index deviennent :

$$
\mathrm{L}=13 \mu, 65 ; l=7 \mu, 57 ; \mathrm{L} / l=1,75
$$

Il résulte de ces premières données biométriquès qu'il existe trois types morphologiques chez Gardia cati :

$1^{\circ}$ Type bréviligne $\mathrm{L} / l=1,5$ à 1,7 rencontré 20 fois sur 100 exemplaires ;

$2^{\circ}$ Type médioligne $\mathrm{L} / l=1,7$ à 1,8 , rencontré 51 fois sur 100 exemplaires, type le plus fréquent par conséquent ;

$3^{\circ}$ Type longiligne $\mathrm{L} / l=$ supérieur à 1,8 , rencontré 39 fois sur 100 exemplaires.

Je ferai successivement l'étude morphologique de ces 3 types. Dans les mensurations qui suivront, exprimées en $\mu$, je désignerai par $L$ la longueur, $l$ la largeur, $a$ le segment compris entre l'extrémité antérieure et le milieu d'une ligne passant par les centres des noyaux, $b$ le segment compris entre ce dernier point et le milieu d'une ligne passant par les deux points correspondant à l'extrémité distale de la portion intra-cytoplasmique des flagelles latéraux postérieurs, $c$ le segment compris entre ce dernier point et l'extrémité postérieure.

$1^{\circ}$ Type bréviligne : $\mathrm{L} / l=1,5$ à 1,7 (fig. 1 ).

Parmi les 20 exemplaires de ce type étudiés, j'ai noté 13 fois de

(1) Les chiffres pour 50 exemplaires communiqués par moi à la Société de Biologie étaient : $\mathrm{L}=13 \mu, 6 ; l=7 \mu, 7 ; \frac{\mathrm{L}}{l}=1,76$. 
grands trophozoïtes globuleux, dont voici les principaux caractères morphologiques :

Mensurations. - Ces mensurations portent sur 10 exemplaires:

\begin{tabular}{|c|c|c|c|c|c|c|c|c|c|}
\hline & \multirow{2}{*}{ L } & \multirow{2}{*}{$l$} & \multirow{2}{*}{$a$} & \multirow{2}{*}{$b$. } & \multirow{2}{*}{$c$} & \multicolumn{2}{|c|}{ Noyacx } & \multicolumn{2}{|c|}{ Crtostome } \\
\hline & & & & & & Longueur & Largeur & Longueur & Laroeur \\
\hline Moyenne... & 14,7 & 9,3 & 4,7 & 6 & 4 & 3,12 & 1,9 & 8,5 & 6 \\
\hline
\end{tabular}
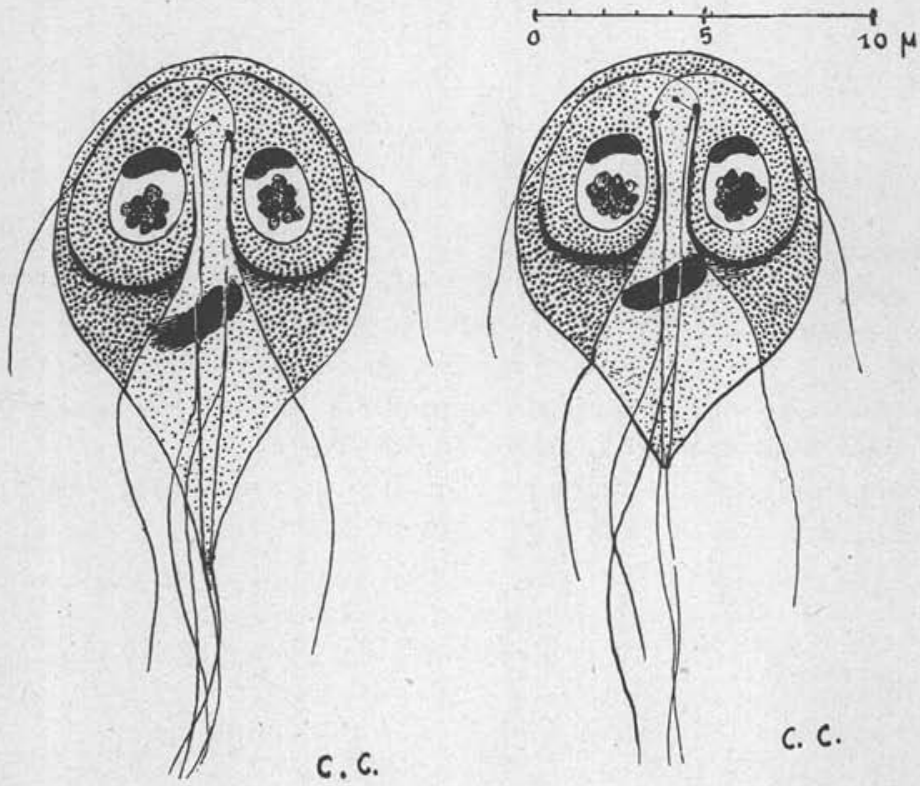

Fıc. 1. - Giardia cati, type bréviligne (flagelles écourtés). $\frac{\mathrm{L}}{1}=1,5$ à 1,7 .

Structure. - Les noyaux sont globuleux, la chromatine est généralement répartie de la façon suivante : au pôle antérieur du noyau, calotte de chromatine assez volumineuse, mais moins importante que chez les formes médiolignes; dans la région centrale du noyau, gros nucléole polylobé ou gros grains chromatiques plus ou moins confluents. Le bord inférieur du noyau affleure parfois le bord inférieur du cytostome.

Les corps parabasaux paraissent généralement fusionnés; ils sont volumineux, transversaux, légèrement obliques, généralement, 
mais non constamment orientés de haut en bas, et de gauche à droite.

Le bord postérieur du péristome est très épais, très sidérophile et entouré de fines granulations sidérophiles très denses.

Parmi les formes brévilignes, j’ai noté, 7 fois sur 20 exemplaires, des trophozoïtes de petite taille sans corps parabasaux, dont les caractères morphologiques sont les suivants :

Mensurations. - Ces mensurations portent sur 7 exemplaires :

\begin{tabular}{|c|c|c|c|c|c|c|c|}
\hline \hline & $\mathrm{L}$ & & & $a$ & $b$ & $c$ & \multicolumn{2}{c|}{ Noyaux } \\
\hline Moyenne...... & 12 & 7,5 & 4 & 4,7 & 3,3 & 2,7 & 1,6 \\
\hline
\end{tabular}

Structure. - La chromatine du noyau présente une répartition générale, semblable à celle décrite pour la forme précédente, mais la chromatine centrale est répartie en grains moins confluents et plus fins. Les corps parabasaux sont absents. Le bord postérieur du cytostome est beaucoup moins épais que dans la forme précédente.

2 . Type médioligne : $\mathrm{L} / l=1,7$ à 1,8 (fig. 2 ).

Ce type est observé dans 51 pour 100 des cas ; c'est donc, je le rappelle, la forme la plus fréquemment observée.

Mensurations. - Ces mensurations portent sur 10 exemplaires:

\begin{tabular}{|c|c|c|c|c|c|c|c|c|c|}
\hline & $\mathrm{L}$ & $l$ & $a$ & $b$ & $c$ & \multicolumn{2}{c|}{ Noyaux } & \multicolumn{2}{c|}{ Cxтostome } \\
\hline Moyenne... & 13,4 & 7,6 & 4 & 5,4 & 4 & 2,6 & 1,7 & 7 & 6 \\
\hline
\end{tabular}

Structure. - La structure des noyaux est la suivante :

Chez les formes dont $\mathrm{L} / \mathrm{l}$ tend vers 1,8 , formes de taille généralement médiocre, le pôle antérieur du noyau est le plus souvent occupé par une calotte de chromatine volumineuse pouvant occuper le $1 / 3$ du noyau; la portion immédiatement sous-jacente à ce nucléole est anhiste, le reste du noyau est occupé le plus souvent par de fines granulations de chromatine, organisées en amas juxtaposés. Parfois le nucléole est situé, non au pôle antéricur, mais latéralement; il est toujours en contact par sa convexité avec la concavité de la membrane nucléaire. Ces dispositions sont 
généralement, mais non toujours, symétriques dans les deux noyaux. Les nucléoles peuvent cependant exceptionnellement être situés, l'un au pôle antérieur, l'autre au pôle postérieur, chez un même parasite. Dans certains parasites, enfin, chaque noyau peut contenir deux nucléoles situés aux pôles opposés.

Chez les formes dont $\mathrm{L} / l$ varie de 1,76 à 1,7 , formes de plus grande taille en général que les précédentes, la répartition de la chromatine est le plus souvent semblable à celle qui a été notée pour les grandes formes brévilignes. Les granulations chromatiques, dont la
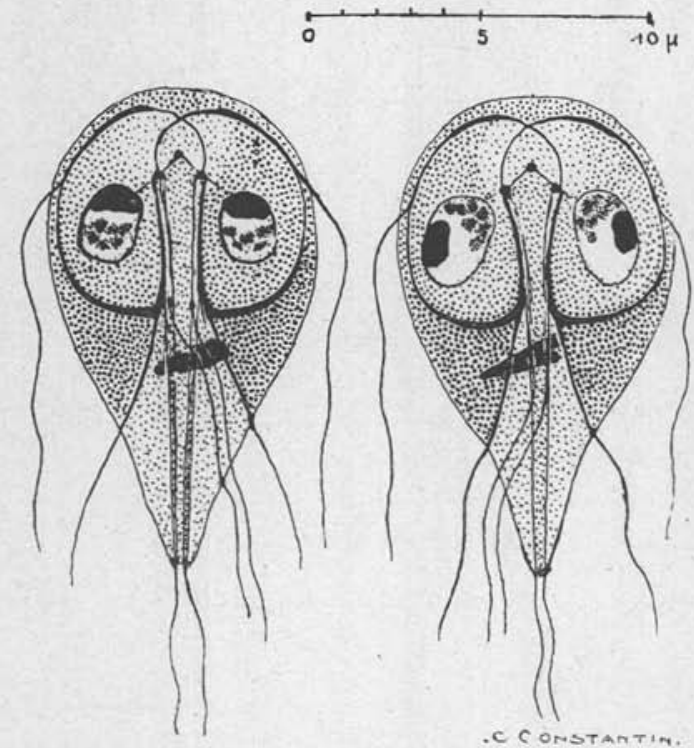

Fıt. 2. - Giardia cati, type médioligne (flagelles écourtés). $\frac{\mathrm{L}}{\mathrm{l}}=1,7$ à 1,8 .

confluence constitue un gros nucléole central, sont généralement plus fines et mieux individualisées, mais on observe toutes les transitions entre le gros nucléole polylobé des grandes formes brévilignes et les amas de fines granulations décrites chez les formes médiolignes de taille médiocre.

Les corps parabasaux (50 exemplaires) sont absents dans $220 / 0$ des cas, punctiformes dans $180 / 0$ des cas, en virgule orientée de haut en bas et de gauche à droite dans $320 / 0$ des cas, en virgule orientée de haut en bas et de droite à gauche dans $280 / 0$ des cas ; ils sont fusionnés dans $970 / 0$ des cas environ. Les petites formes ont des corps parabasaux très grêles ou n'en présentent point. Le 
bord inférieur du péristome a généralement une épaisseur proportionnelle à la taille des corps parabasaux et à la condensation de la chromatine dans les noyaux.

$3^{\circ}$ Type longiligne : $\mathrm{L} / l=1,8$ à 2,16 (fig. 3 ).
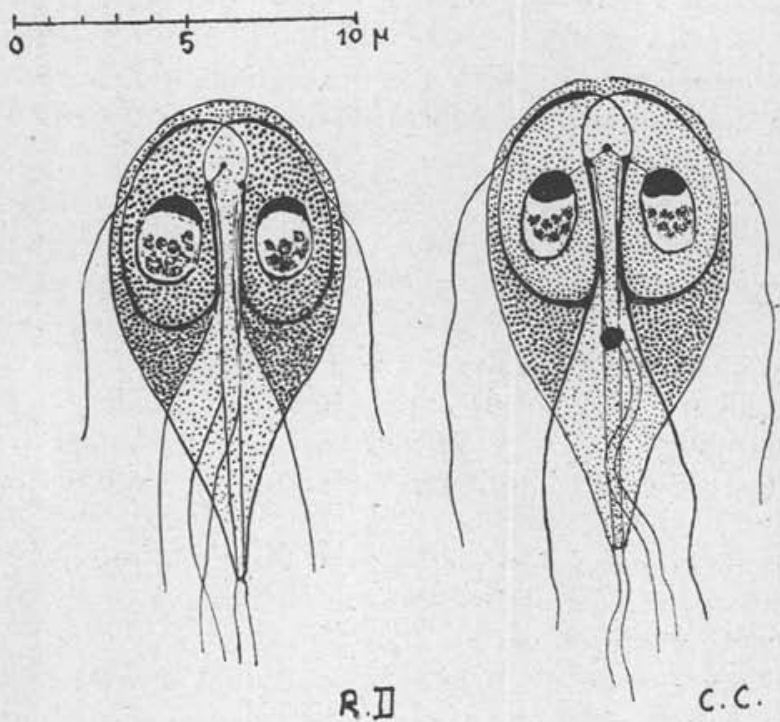

Fı́. 3. - Giardia cati, type longiligne (flagelles écourtés). $\frac{\mathrm{L}}{\mathrm{l}}=1,8$ à 2,16 .

Mensurations. - Ces mensurations portent sur 10 exemplaires:

\begin{tabular}{|c|c|c|c|c|c|c|c|c|c|}
\hline & \multirow{2}{*}{ L } & \multirow{2}{*}{. $t$} & \multirow{2}{*}{$a$} & \multirow{2}{*}{$b$} & \multirow{2}{*}{$c$} & \multicolumn{2}{|c|}{ Noyatx } & \multicolumn{2}{|c|}{ Cytostome } \\
\hline & & & & & & Longueur & $\widehat{\text { Largeur }}$ & Longueur & Laryeur \\
\hline Moyenne... & 13,6 & 7,1 & 4,1 & 5,5 & 4 & 2,3 & 1,25 & 6,2 & 6 \\
\hline
\end{tabular}

Structure. - Le noyau, chez 9 exemplaires sur 10, présente la structure décrite chez les formes médiolignes dont $L / l$ tend vers 1,8 . Cependant, la calotte chromatique située au pôle antérieur est beaucoup moins haute et les granulations chromatiques beaucoup plus fines. Une fois sur 10 exemplaires, le noyau présentait un petit nucléole central à l'exclusion de toute autre granulation chromatique. 
Les corps parabasaux sont absents 9 fois sur 10 exemplaires et lorsqu'ils existent, ils sont punctiformes ou très grêles.

Le bord inférieur du cytostome est généralement moins épais que dans les formes brévilignes et médiolignes.

$$
* *
$$

Lorsqu'on fait une étude comparée de ces divers types morphologiques, un peu schématisés d'ailleurs puisqu'ils représentent un maximum de fréquence ou une moyenne, on est amené à faire les constatations suivantes :

Les formes longilignes ne diffèrent des formes médiolignes que par leur moindre largeur moyenne (longilignes 7,1, médiolignes 7,6), leurs noyaux plus petits (longilignes : $2,3 \times 1,5$, médiolignes : $2,6 \times 1,7$ ), leur moindre richesse en chromatine, l'absence ou le très faible développement des corps parabasaux.

Les longueurs moyennes sont à peu près égales (longilignes : 13,6, médiolignes : 13,4$)$, ce qui ne doit pas surprendre si l'on veut bien admettre que les longilignes résultent de la scissiparité des médiolignes.

La structure nucléaire des deux types morphologiques est semblable dans 3 exemplaires sur 10 . On trouve des formes limites de structure identique.

Les grandes formes brévilignes ne diffèrent des médiolignes que par leur plus grande taille, leur longueur moyenne plus élevée (brévilignes : 14,7, médiolignes : 13,4), leur largeur moyenne plus élevée (brévilignes : 9,3, médiolignes : 7,6 ), la plus grande taille des noyaux (brévilignes : $3,12 \times 1,9$, longilignes : $2,6 \times 1,7$ ), leur richesse et leur condensation plus grande en chromatine, le très grand développement des corps parabasaux qui est généralement proportionnel à la taille. Les structures générales et nucléaires des deux types morphologiques sont semblables, dans 8 exemplaires sur 10. En résumé, les brévilignes conduisent aux médiolignes, qui conduisent aux longilignes.

Ces différentes formes appartiennent-elles à la même espèce ? J'estime qu'on doit répondre par l'affirmative, étant donnée l'identité du plan de composition des noyaux et l'existence des formes de transition ou de formes similaires pour deux types différents.

Comment interprêter ces différents aspects morphologiques ? Quatre données paraissent les expliquer :

$1^{\circ}$ Les formes résultant d'une division par scissiparité d'un trophozoïte, ont une longueur égale à ce trophozoïte, mais une largeur moindre. 
$2^{\circ}$ La condensation et la richesse en chromatine sont généralement proportionnelles à l'âge des formes végétatives.

$3^{\circ}$ La taille des corps parabasaux est généralement proportionnelle à la taille et à la largeur des formes végétatives et, par conséquent, à leur âge. A cet égard, les figures données par Lavier chez G. simoni sont particulièrement démonstratives.

$4^{\circ}$ Les formes récemment enkystées que l'on reconnait à leur structure qui est celle d'une forme végétative arrondie ayant perdu ses flagelles, ont un noyau riche en chromatine et des corps parabasaux volumineux.

Si l'on admet ces 4 points, dont les 3 derniers ne peuvent être définitivement établis que par la culture de Giardia, non encore réalisée, les formes longilignes à corps parabasal absent ou de petite taille, à faible largeur moyenne et à noyau relativement pauvre en chromatine, doivent être considérées comme des formes résultant d'une division récente; les formes médiolignes doivent être considérées comme des formes plus évoluées, et les grandes formes brévilignes de grande taille et de grande largeur, à noyau riche en chromatine condensée et à corps parabasaux volumineux, apparaissent comme des trophozoïtes àgés au stade prékystique. Quant aux petites formes brévilignes, d'ailleurs les moins nombreuses $(70 / 0)$, il est difficile de les interpréter. L'hypothèse du désenkystement occasionnel dans l'intestin d'un hôte, de kystes formés dans cet hôte, a été envisagée par Perroncito (1902) et formulée par Simon (1921), par moi-même (1921-1923), et, tout récemment, par Hegner (1925) ; on pourrait supposer, avec de grandes réserves, que les petites formes brévilignes ne possédant pas de corps parabasal, et ayant un noyau pauvre et peu condensé en chromatine, sont des formes jeunes et récemment désenkystées.

\section{Recherches expérimentales}

$1^{\circ}$ Inoculation au cobaye. - Deux cobayes de six semaines reconnus non infectés par Giardia après épreuve de trois semaines ont été inoculés "per os " avec de nombreux kystes vivants (épreuve de l'éosine) de Giardia du chat $\mathrm{n}^{\circ} 2-1$. Les animaux ne s'infectèrent pas ; autopsie négative au seizième jour.

$2^{\circ}$ Inoculation a la souris. - Deux souris de un mois non infectées par Giardia ont été inoculées " per os " et à deux reprises avec de nombreux kystes vivants de Giardia provenant du chat $\mathrm{n}^{\circ} 1-1$; résultat négatif, au quinzième jour ; autopsie négative. 
$3^{\circ}$ Inoculation au chien. - Un chiot de deux mois non infecté par Giardia, inoculé " per os " avec des kystes vivants provenant du chat $\mathrm{n}^{\circ} 2-1$, suivi pendant un mois, ne s'infecta pas ; l'autopsie n'a pu être faite.

\section{Diagnose différentielle}

La giardie que nous avons étudiée peut-elle être identifiée à une espèce précédemment établie ?

\section{Poissons}

Giardia denticis Fantham, 1919, du sang de Dentex argyrozona, ne doit pas être retenue étant donnés l'éloignement zoologique des hôtes et leur répartition naturelle et géographique.

\section{Batraciens}

Giardia agilis Kunstler, 1881, est distincte morphologiquement de la giardie du chat comme de toutes les autres giardies d'ailleurs. Kunstler et Gineste (1907), Reuling et Rodenwaldt (1921) en font un sous-genre distinct.

\section{Reptiles}

Giardia varani Lavier, 1923, observée chez un saurien de l'Afrique occidentale, Varanus niloticus, est morphologiquement distincte de la giardie du chat. $G$. varani : moyennes, $\mathrm{L}=16, l=8$, $\mathrm{L} / l=2$. Corps parabasaux bacillaires et grêles. En outre, l'éloignement zoologique des hôtes et leur répartition géographique différente permettent d'écarter cette espèce.

\section{Oiseaux}

Parmi les giardies décrites chez les oiseaux : G. ardeæ Noller, 1920, du héron (Ardea cinerea et Ardetta minuta); Giardia sp. Kotlan, 1923, de l'avocette (Recurvirostra avocetta); Giardia sp. Kotlan, 1923, de la pie-grièche (Lanius callurio); Giardia sp. Hegner, 1925, de Nycticorax nycticorax nævius; Giardia sp. Hegner, 1925, de Ardea herodias, n'ont certainement aucun rapport de spécificité avec la giardie du chat. Leur morphologie (type G. muris) en est absolument distincte, outre que l'éloignement zoologique et la répartition géographique des hôtes permettent peu de retenir 
cette hypothèse. G. sanguinis Gonder, 1911, de l'élanion blanc (Elanus coruleus), bien que se séparant du type morphologique des autres espèces de Giardia d'oiseaux décrites jusqu'à ce jour, est nettement distincte morphologiquement de la giardie du chat ; G. sanguinis : moyennes, $\mathrm{L}=12 ; l=8 ; \mathrm{L} / l=1,5$; structure nucléaire différente.

\section{Mammifères}

G. muris Grassi, 1879, de la souris est morphologiquement distincte de la giardie du chat: G. muris : moyennes, $L=9,75$; $l=7,26 ; \mathrm{L} / l=1,35$. Structure nucléaire distincte. En outre, je n'ai pas réussi à infecter 2 souris avec des kystes de la giardie du chat.

G. microti Kofoid et Christiansen, 1915, de Microtus californicus est morphologiquement distincte de la giardie du chat : G. microti: moyennes, $\mathrm{L}=11,06 ; l=6,79 ; \mathrm{L} / l=1,66$. Structure nucléaire distincte.

G. simoni Lavier, 1924, du rat d'égout, Epimys norvegicus, avec les dimensions moyennes suivantes : $\mathrm{L}=17 ; l=9 ; \mathrm{L} / l=1,90$; se rapproche morphologiquement des grandes formes brévilignes de la giardie du chat, mais la longueur plus élevée ( $G$. simoni : L, maximum de fréquence $=17 ; G$. cati: $\mathrm{L}$, maximum de fréquence $=$ 13 et maximum noté 16,5 ) et la structure nucléaire distincte (il n'existe pas de calotte de chromatine au pôle antérieur du noyau chez $G$. simoni) de G. simoni ne permettent pas d'identifier la giardie du chat à cette espèce.

G. pitymisi Splendore, 1920, du campagnol, probablement identique à Giardia sp. Lavier, 1921, de Microtus arvalis, d'après Lavier, est de taille très inférieure à la giardie du chat, Giardia sp. Lavier : moyenne, $\mathrm{L}=10, ; l=6,3$, et a une structure nucléaire différente.

G. viscacix Lavier, 1923, de la viscache (Viscacia viscacia) présente une longueur et une largeur moyennes plus grandes $(L=16$; $l=8,5)$ que celles de la giardie du chat, la disposition de la chromatine dans le noyau est différente, il n'existe pas en particulier de volumineuse calotte de chromatine au pôle antérieur mais un simple épaississement de la membrane nucléaire ou un granule de chromatine à la partie antéro-interne. Enfin la répartition géographique de la viscache et du chat domestique ne coïncident pas; la giardie du chat est donc distincte de $G$. viscacia.

G. duodenalis Davaine, 1875, du lapin (Lepus cuniculus) présente une structure nucléaire et des dimensions différentes de la giardie du chat : $G$. duodenalis : moyennes $\mathrm{L}=15,8 ; l=9,1$. 
G. caviæ Hegner, 1923, du cobaye (Cavia cobaya) présente une structure nucléaire, des dimensions et des proportions très différentes de celles de la giardie du chat; en outre, j'ai inoculé sans succès " per os " des kystes de la giardie du chat à deux jeunes cobayes de 6 semaines.

G. capræ Nieschulz, 1923, de la chèvre, présente des analogies morphologiques avec la giardie du chat. La longueur $\mathrm{L}=13,5$, la largeur $l=7,5$, le rapport $L / l=1,8$ sont voisins de ceux observés chez la giardie du chat et le noyau a une structure semblable à celle du noyau de certaines formes longilignes et médiolignes de la giardie du chat; en particulier, la calotte chromatique notée chez cette dernière se retrouve chez G. capræ. Par contre, Nieschulz n'a pas noté des types morphologiques différents et des structures nucléaires correspondant à celle des grandes formes brévilignes de la giardie du chat; en outre, les corps parabasaux de G. capræ sont longs et grêles. La différenciation biologique est nécessaire, mais il est probable, étant donnés les régimes alimentaires très différents du chat et de la chèvre d'une part, et, d'autre part, les rares relations biologiques de la chèvre et du chat, que les deux espèces de giardies sont distinctes.

G. bovis Fantham, 1921, du bœuf, est morphologiquement distincte de la giardie du chat $(\mathrm{L}=23 ; l=13 ; \mathrm{L} / l=1,77)$.

G. equi Fantham, 1921, du cheval, est morphologiquement distincte $: \mathrm{L}=20 ; l=10 ; \mathrm{L} / l=2$.

G. canis Hegner, 1922, du chien domestique (Canis familiaris), est plus large ; le rapport $\mathrm{L} / l=1,62$, index très supérieur à celui de la giardie du chat ; la structure nucléaire est distincte et je n'ai pu inoculer un jeune chien de 2 mois avec des kystes de la giardie du chat.

G. intestinalis (Lambl, 1859), de l'homme, est plus longue que la giardie du chat $(G$. intestinalis : $\mathrm{L}=13,7)$ et de largeur égale ; le rapport $\mathrm{L} / l=1,84$ est donc supérieur à celui de la giardie du chat ; en outre, on n'observe pas dans le noyau, chez G. intestinalis, la calotte polaire de chromatine observée chez la giardie du chat. Enfin, alors que l'inoculation positive de chat à chat de la giardie du chat est très facile, comme je l'ai établi plus haut, l'inoculation de $G$. intestinalis au chat est négative dans la généralité des cas. Les deux espèces doivent être, dans l'état présent de nos connaissances, considérées comme distinctes.

Il résulte de cette étude comparée que la giardie du chat doit être considérée comme une espèce distincte. 


\section{Identité de G. cati et de G. felis}

Il me reste à rechercher si Giardia cati Deschiens, 1925, et Giardia felis Hegner, 1925, constituent une même espèce ou deux espèces distinctes :

La longueur de Giardia cati : $\mathrm{L}=13$ est légèrement supérieure à celle de Giardia felis : $\mathrm{L}=12,66$, mais cet écart de $0 \mu, 33$ environ peut fort bien être de l'ordre des variations morphologiques d'une même espèce.

La largeur de Giardia cati : $l=7,5$ est supérieure de près de $1 \mu$ à celle de Giardia felis : $l=6,6$; il y a donc là un écart important, mais en me reportant à mes tables de mensurations, j'ai noté que les formes longilignes présentaient dans 2 pour 10 des cas environ une largeur comprise entre $6 \mu$ et $6 \mu, 7$ avec une longueur correspondante de 12 à $13 \mu$, ce qui répond exactement aux dimensions indiquées par Hegner.

Le rapport longueur sur largeur est moins élevé chez Giardia cati: $\mathrm{L} / l=1,76$ que chez Giardia felis $: \mathrm{L} / l=1,92$; mais, chez les formes longilignes de Giardia cati le rapport L/l est compris entre 1,8 et 2 avec maximum de fréquence à 1,9 .

Hegner n'indique pas dans son texte les dimensions des noyaux observés chez Giardia felis, mais lorsqu'on les mesure sur le " canon " de Giardia felis dessiné par lui, on obtient : longueur $2 \mu, 3$, largeur $1 \mu$, 3. Ces chiffres correspondent aux dimensions des noyaux des formes longilignes de Giardia cati : $2,3 \times 1,24$.

La structure nucléaire figurée sur un dessin à la chambre claire donné par Hegner répond à un type observé chez les formes médiolignes et longilignes de Giardia cati, bien que ce type ne soit pas le plus commun. La calotte chromatique du pôle antérieur est nette sur l'exemplaire de Giardia felis figuré bien que moins développé que dans la majorité des exemplaires de Giardia cati.

Les corps parabasaux en virgule figurés sur le "canon " et le dessin de Giardia felis d'une part, et, d'autre part, ceux de certaines formes médiolignes et longilignes de Giardia cati sont tout à fait semblables, avec eette réserve que j'ai trouvé les corps parabasaux de Giardia cati fusionnés dans la majorité des cas, alors que les deux dessins de Hegner les figurent isolés.

Reste la comparaison des segments $a, b, c$, on note (10 exemplaires mesurés) :

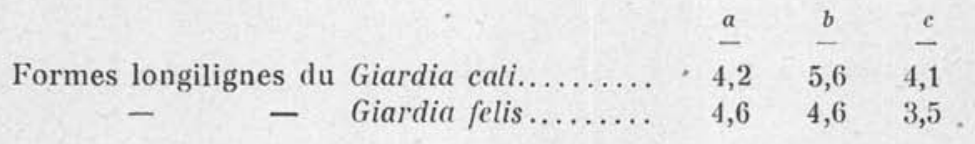


Les dimensions du segment $b$ ont done une différence de $1 \mu$ entre elles, mais cette différence, sur un point secondaire, he saurait être retenue contre la concordance de tous les autres caractères, et d'ailleurs on note chez certaines formes longilignes de Giardia cati répondant aux schémas de Hegner, en longueur, largeur et proportion : $a=4, b=4,5, c=3,5$.

Je pense donc que Giardia cati et Giardia felis appartiennent à la même espèce et que le savant professeur de Baltimore a observé des souches de giardies du chat particulièrement riches en formes longilignes de petite taille. Cette question peut d'ailleurs être éclaircie par la comparaison de préparations de Giardia cati et de Giardia felis. C'est pourquoi j'ai adressé à M. le professeur Hegner l'une de mes préparations de Giardia cati.

\section{RÉsumé}

La giardie du chat domestique est morphologiquement distincte des espèces précédemment décrites ; on distingue chez cette giardie un type bréviligne, un type médioligne et un type longiligne.

Les kystes de la giardie du chat infectent le chat expérimentalement, par contre deux jeunes cobayes, deux jeunes souris et un jeune chien n'ont pu être infectés dans les mêmes conditions. La giardie du chat doit donc être considérée comme une espèce distincte que j'ai décrite sous le nom de Giardia cati.

La comparaison de Giardia cati Deschiens et de Giardia felis Hegner, décrite par cet auteur, chez le chat domestique, dans un mémoire ultérieur à ma première note, indique que Giardia cati et Giardia felis sont presque certainement une même espèce et que Giardia felis répond aux formes longilignes de petite taille de Giardia cati.

\section{Bibliographie}

Bensex. - Bau und Arten der Gattung Lamblia. Zeitschrift f. Hyg. und InfektionsKrankh., LXI, 1908, p. 109.

Davaine. - Monadiens. Dict. Encycl. des Sc. Méd., série 2, IX, 1875, p. 115-130.

Deschiens (R.). - Les entérites à Giardia (Lamblia). Thèse de la Faculté de Médecine de Paris, 1921, p. 51 et 70.

- Variations tinctoriales et morphologiques de Giardia intestinalis. Bull. Soc. pathol. exot., XVI, 1923, p. 323.

- Giardia cati n. sp. du chat domestique. C. R. Soc. de Biol., XCII, 9 mai 1925 , p. 1271. 
Doflers, - Lehrbuch der Protozoenkunde, 1916.

Fantham (H.-B.). - Some parasitic protozoa found in South Africa (II). South Africa Jl. of Sc., Johannesburg, XVI, octobre-décembre 1919.

Some parasitic protozoa found in South Africa (IV). Ibid., 1921.

Gonder. - Lamblia sanguinis n. sp. Arch. f. Protistenk., XXI, 1910, p. 209.

(iondon (R.-M.) et Young (C.-J.). - Parasites of dogs and cats in Amazonas. Ann. Trop. Med. and Parasit., XVI, 1922, p. 297-300.

Grassı (B.) et Schewiakoff (W.). - Beitrag zur Kenntniss des Megastoma entericum. Zeitschr. wiss. Zool., XLVI, 1888, p. 143-154.

Hegner (R.-W.). -- A comparative study of the Giardia living in man. rabbit and dog. Am. Journ. of Hyg., II, juillet 1922, p. 442-454.

The systematic relationship of Giardia lamblia Stiles, 1915, from man and Giardia agilis Kunstler, 1882, from the Tadpole. Am. Jonrn. of Hyg., II, f. 4,1922 , p. $435-441$.

Giardia from wild rats and mice and Giardia cavia n. sp. from the Guinea pig. Proc. Soc. of Hyg., 16 février 1923, in Am. Jl. of Hyg., III, mai 1923, p. $345-349$.

Giardia and Chilomastix from monkeys, Giardia from the wild cat and Balantidinm from the sheep. The $J l$. of Parasit., XI, septembre 1924, p. 75.

Giardia felis n. sp. from the domestic cat and giardias from birds. Am. $J l$. of Hyg., V, mai 1925, p. 258-273.

Hogue (M.-J.) et VAN WinkLe. - The effect of carbone tetrachloride on intestinal protozoa. Am. Jl. of 7 rop. med., III, mai 1923, p. 197-202.

Janowski. - Flagellaten in der menschlichen Faeces. Zeit. f. klin. Med., XXXI, 1897, p. 442-492.

Koford et Christiansen. - On Giardia microti sp. nov. from the meadow mouse. Univ. California Publ. Zool., XVI, 1925, p. 23-29.

KoFord. - On binary and multiple fission in Giardia muris Grassi. Univ, California Publ. Zool., 1915, p. 30-54.

The biological and medical signifiance of the intestinal flagellates. Proc. pan. Am. Scient. Congr. Washington, X, 1917, p. 546-565.

Kotláx (A.). - Giardien (Lamblien) in Vögeln. Centralbl. f. Bakt. Orig., XC, 1923, p. 24-28.

- Zur Kenntnis der Darmflagellaten aus der Hausente und anderen Wasservögeln. Centrabl. f. Bakt. Orig., XC, 1923, p. 24-28.

Kunstrer et Gineste. - Megastoma, Lamblia ou Giardia. Bull. Soc. Zool. de France, XXXII, 1907, p. 28.

Lavien (G.). - Flagellés parasites intestinaux du campagnol indigène, Microlus arvalis. Bull. Soc. path, exot., XIV, 1921, p. 710-717.

Sur deux espèces nouvelles du genre Giardia: G. viscacia de la viscache (Viscacia viscacia) et $G$. varani du varan (Varanus niloticus). Ann. de Parasitol., I, 1923, p. 147-154.

- Deux espèces de Giardia du rat d'égout parisien (Epimys norvegicus). Ann. de Parasilol., II, 1924, p. 161-168.

Nreschelz (O.). - Een geval van Giardia infectie bij een kalf. Tijdsch. Diergeneesk., Utrecht, L, 1923, p. 733-735.

- Giardia capre n. sp. en Entamœba sp., nieuve darmparasiten van de geit. Tijdsch. v. Diergeneesk. Utrecht, L, 1923, p. 780-783.

- Uber den Bau von Giardia capre mihi. Arch. f. Protistenkunde, XLIX, novembre 1924, p. 278-286.

Noller (W.). - Kleine Beobachtungen an parasitischen Prozotoen. Arch. f. Protistenkunde, XLI, 1920, p. 169-189. 
Perroncito. - Incapsulmento del Megastoma inlestinale. Gior della R. Accad. med. Torino, 13 mai 1887.

Realing (I.) et Rodenwal.t (E.). - Giardia ? Lamblia? Arch.f. Protistenk., XLII, 3,1921 , p. $337-346$.

Sison (Ch.-E.). - Giardia enterica : A parasitic intestinal flagellate of man. Am. Jl. Hyg., IV, 1921, p. 440-491.

- A critique of the supposed rodent origin. of human giardiasis. Am. $J$. Hyg., II, juillet 1922, p. 406-433.

SPlendore. - Sui parassili delle arvicole. Annali d'Igiene, XXX, 9, 1920, p. 561.

Laboraloire de Parasilologie de la Facullé de médecine de Paris. 